\title{
A Gamified Mobile-Based Approach with Web Monitoring for a Crowdsourcing Framework Designed for Urban Problems Related Smart Government: A Case Study of Chiang Mai, Thailand
}

\author{
https://doi.org/10.3991/ijim.v13i12.10989
}

Kitti Puritat

Chiang Mai University, Chiang Mai, Thailand

kitti.p@emu.ac.th

\begin{abstract}
Crowdsourcing in smart cities has rapidly grown with the buildup of the internet of things which has enabled citizens who are similarity thinking in the society to become increasingly connected with one another delivering information to the government in order to plan the city. This article proposes a gamified crowdsourcing framework for smart governments to solve urban problems. It could help government officers to organize the deliveries regarding the city's characteristics. We applied game elements from the Gamification Concept in a mobile application in order to engage the citizens in our framework. We evaluated the framework for 2 months which had 548 citizens according to system data. According to our crowd sourcing project the most important issues that Chiang Mai is facing are pollution and traffic jams.
\end{abstract}

Keywords-Crowdsourcing, Gamification, Smart government, Smart city, Analysis Visualization, Mobile application

\section{Introduction}

During the past two decades, digital technology continued to be more broadly available and implemented in terms of city management. Hence, it is used to solve the problem of increasingly sprawling, populated cities, with heavy traffic and the ensuing problems of environmental degradation. Most of the researchers call the concept Smart Cities as transformation initiatives focus on information communication technologies (ICT), knowledge resources, social cohesion, and social infrastructure for economic regeneration, infrastructure management, and better city administration [1]. Since 2003, the first idea of smart cities in Thailand has been introduced, while the idea was first implemented by the Ministry of Information and Communication Technology (MICT). Then, it later changed its name in 2016 to the Ministry of Digital Economy and Society (MODES) in order to facilitate the spread of digital technology as an agent of national economic development .After that, MODES became the main body to drive the idea of implementation of the smart cities concept in Thailand. This research aims to propose a smart crowdsourcing framework for collecting information 
using the gamification approach focusing on Chiang Mai province. The input data is based on the information obtained from those citizens who used our application based on the concept of the popular game called Pokemon go. In2016, Niantic proposed the mobile application game Pokemon GO. The concept of the game is to allows players to collect and search for Pokemons in the physical environment using geo-location and to catch the Pokemons in an augmented reality based on the real environment. The system proposed in this research uses data from geo-location in crowdsourcing in order to collect information via mobile data collection by indigenous people. The collected information can be related to issues such as broken public facilities, traffic jams, accidents crimes and other major problems in Chiang Mai, e.g. air pollution with pm 2.5 etc. When the information is collected, the government officer can handle or solve the problems and the governments could set the rules on creating the policies relevant to the cities' needs.

The purpose of this research consists of the following:

1) Proposing the framework of smart crowdsourcing in order to collect the information on problems around Chiang Mai city.

2) Collecting real-time Geo-location data for visualization allowing map-based reporting for government officers.

3) Proposing a mobile application applying the concept of gamification in order to engage citizens in providing information for the government.

4) Proposing the application of clustering algorithms to cluster the map points converting them to a map in order to provide easy-to-understand input for the decisionmaking of government officer.

The structure of this paper consists of 5 sections as follows: section 1 is the introduction and section 2 explains the definitions and related works. Section 3 proposes the architecture of the system. Section 4 shows the method of clustering map points to an organized smart map and section 5 shows the results and the system performance evaluation. Lastly, we identify the limits of our current work and set the future directions of our framework.

\section{Definition and Related Work}

The concept of crowdsourcing is always based on utilizing the participation in projects which need a huge number of citizens to solve certain problems together. It could help managing the cost of their participation in traditional ways, by outsourcing or 'in-house' which is not effective. One of the most popular definition of crowdsourcing is "channeling the experts' desire to solve a problem and then freely sharing the answer with everyone" [12].

Generally, following the idea of [7] the crowdsourcing framework could be roughly separated into four categories depending on the characteristics of the crowdsourced work (Fig. 1). First, crowd processing is the concept of a huge number of homogeneous tasks which recommends those who are interested in contributing to the tasks. Thus the value is depending on each isolated contribution such as Amazon Mechani- 
cal Turk or Galaxy Zoo. Second, crowdsolving is the concept of finding heterogeneous solutions to solve complex problems such as building an academy or Yomken. Third, crowd rating is the concept of collecting opinions and evaluating ideas. For example, the framework to translate a language with improved quality by huge number of homogeneous 'votes' [14]. Finally, the last concept of crowd-creating is to create the solution or idea from heterogeneous contributions. The concept of creation includes knowledge or content such as YouTube or Wikipedia. Our framework implemented both concepts of crowdsolving and crowd-creating in order to provide information and generate ideas from government officers.

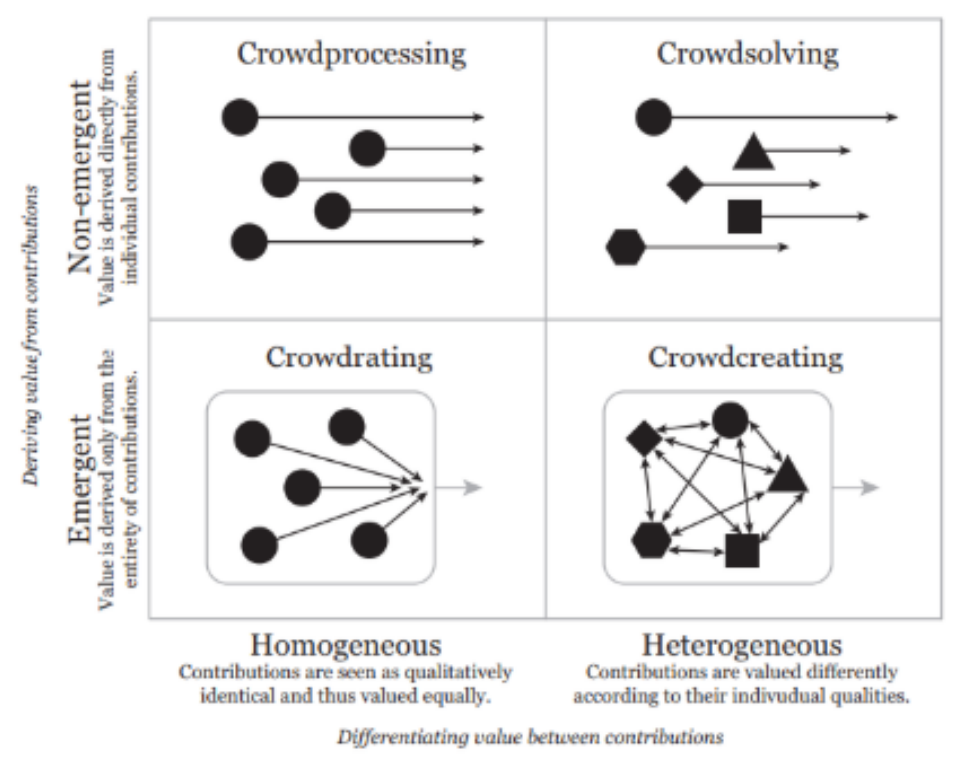

Fig. 1. Four different forms of crowdsourcing contributions[2].

\subsection{Definition of gamification}

Normally, the term Gamification is mostly used in various fields such as business, education, healthcare and marketing defined as "application of game-design elements and game principles in non-game contexts" $[8,10]$. Specifically, we aim to combine the research work of crowdsourcing with Gamification. The main challenge in field of crowdsourcing is to motivate participants to join the activity more frequency. The approach of Gamification applied to crowdsourcing mostly focuses on improving motivation for participation $[13,17]$ and on providing incentive structures $[6,9]$. 


\subsection{Related smart city framework}

The IES smart city framework [16] presented open technological platforms in different cities across Europe. The specific objectives of this framework are linking data management, services and social data mining together. The CityPulse framework [3] tries to connect the service of a distributed system for data analytics, semantic discovery, and social media analytics in implementing large-scale (near-) real-time Internet of Things data. Garuda Smart City Framework [15] proposed standard indicators as the model of smart city for Indonesia including social life, people, governance, economy and technology implementation.

\section{Proposed System Architecture}

The smart city framework aims to collect information from people living in city environment. Actually, there are three types of actors in the framework: people, government officers and the Government CEO. For each actor, we proposed the three system architectures in order to solve the problem for each role Fig 2. Mobile Gamified Crowdsourcing receives environmental problems in the form of city data via mobile application. Users can set up the flag (new problem) or join a flag (problem) by sharing, commenting similar to social media concepts applying the concept of Gamification. The Backend Server has to filter up the problem context or comments which are not appropriate. The data is then proceeded and transferred to a real-time monitor and visualized in a map format. Finally the real-time cloud-based dashboard represents the overview of the problem. Cluster levels can be grouped with specific latitude and longitude in the visualized map. The details of each system will be mentioned below. 


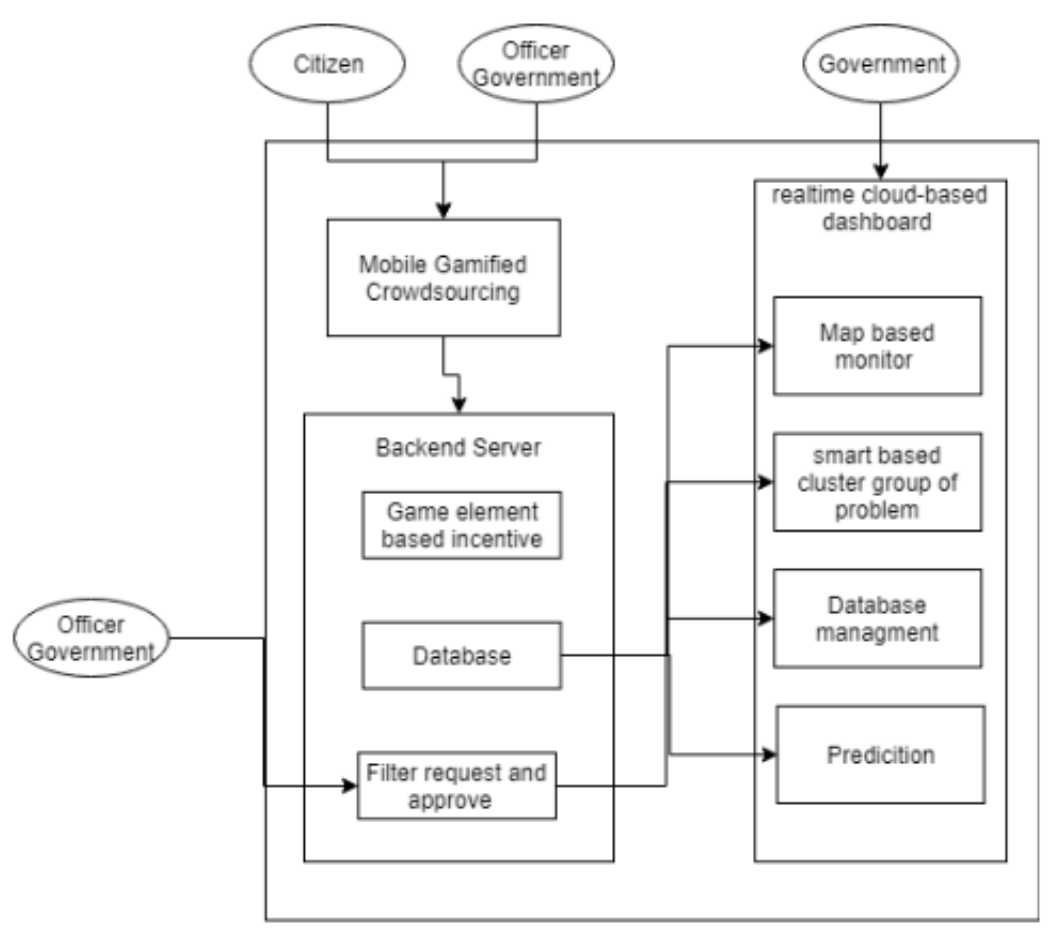

Fig. 2. Proposed system architecture of Gamified crowdsourcing.

\subsection{Mobile gamified crowdsourcing}

Mobile Gamified Crowdsourcing is based on the concept of Gamification with applied game mechanics, game dynamics and game rules in order to create engagement among citizens and provide information for the government. It also uses a Locationbased game where citizens provide real position latitude and longitude for the position in the Mobile Gamified Crowdsourcing. The concept employed the game mechanics from Pokemon GO where the players move around the places where they live. Their smartphones vibrate to let them know when they are near a Flag of problem Fig.3. Once they encounter a Flag of problem, they can address that problem by suggesting a solution, making acomment, by likes or dislikes. 

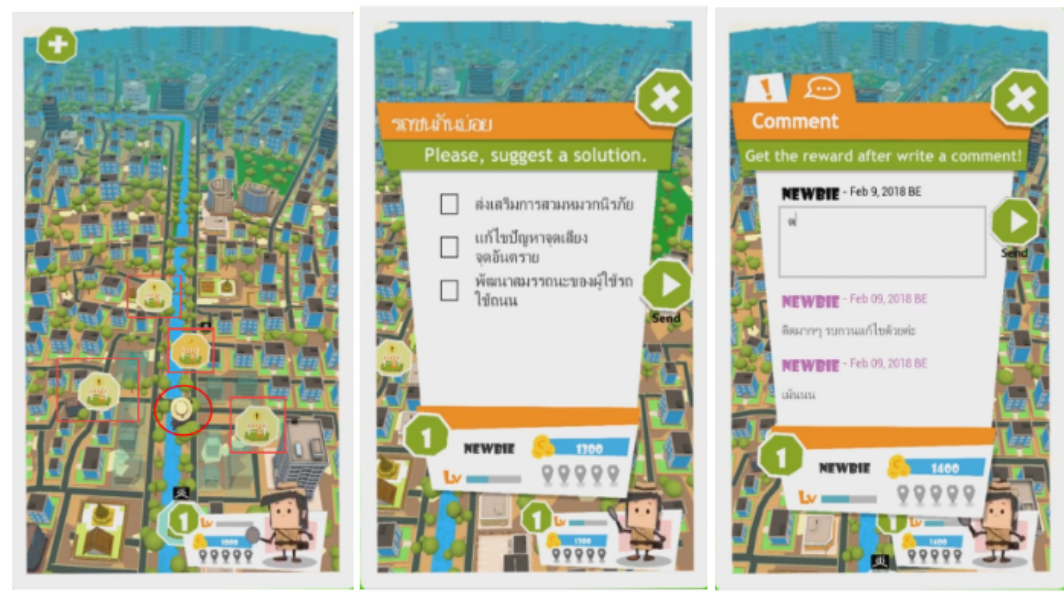

Fig. 3. Game of finding issues; red rectangle is a flag of problem, red circle is the player. Pic 2.Player suggests a solution of a certain flag. Pic 3. Player can comment on the social platform.

However, the key of a successful crowdsourcing system is an active crowd of citizens. In order to motivate citizens to participate the crowdsourcing system, we designed the mobile application based on gamification concept to change the psychological outcome and to motivate citizens to use the crowdsourcing system. For our application, we included the game elements such as ranks, levels, badges and scoring system. Once the player creates or participates a flag of problem they obtain xp scores. If the player gets enough score he or she will be promoted to a higher level and will get a new badge as shown in Table 1 .

Table 1. Game elements implemented in the Gamified Crowdsourcing Framework.

\begin{tabular}{|l|l|l|}
\hline \multicolumn{1}{|c|}{ Game elements } & \multicolumn{1}{|c|}{ Description } & \multicolumn{1}{|c|}{ Applied on crowdsourcing } \\
\hline Levels & $\begin{array}{l}\text { Motivate the players to develop the } \\
\text { skill. For higher levels it may be } \\
\text { more complicated than on lower } \\
\text { levels[19] }\end{array}$ & $\begin{array}{l}\text { We developed a framework where } \\
\text { the player can get to the next level } \\
\text { through a Flag of problem by } \\
\text { participation or creation. This is to } \\
\text { provide engagement when using } \\
\text { the system }\end{array}$ \\
\hline Avatars & $\begin{array}{l}\text { To boost the players' esteem an } \\
\text { avatar represents them and it plays } \\
\text { a very critical role in enhancing } \\
\text { player engagement[18] }\end{array}$ & $\begin{array}{l}\text { The crowd sourcing proposes the } \\
\text { use of avatars for the players. They } \\
\text { can select between man, woman } \\
\text { and can also customize the acces- } \\
\text { sory and clothes }\end{array}$ \\
\hline Social participate & $\begin{array}{l}\text { Social participation can act as a } \\
\text { point of cultural exchange, direct- } \\
\text { ing the flow of information to- } \\
\text { wards particular areas of interest } \\
\text { based on their own social networks } \\
\text { and topics discussed therein to } \\
\text { keep engagement in system[17] }\end{array}$ & $\begin{array}{l}\text { to exchange knowledge and share } \\
\text { the opinion regarding the problem } \\
\text { Ranking serves as a powerful } \\
\text { motivator when players strive for } \\
\text { higher scores [18] }\end{array}$ \\
\hline Leaderboard & $\begin{array}{l}\text { Player can observe their profile } \\
\text { rankings online. }\end{array}$ \\
\hline
\end{tabular}



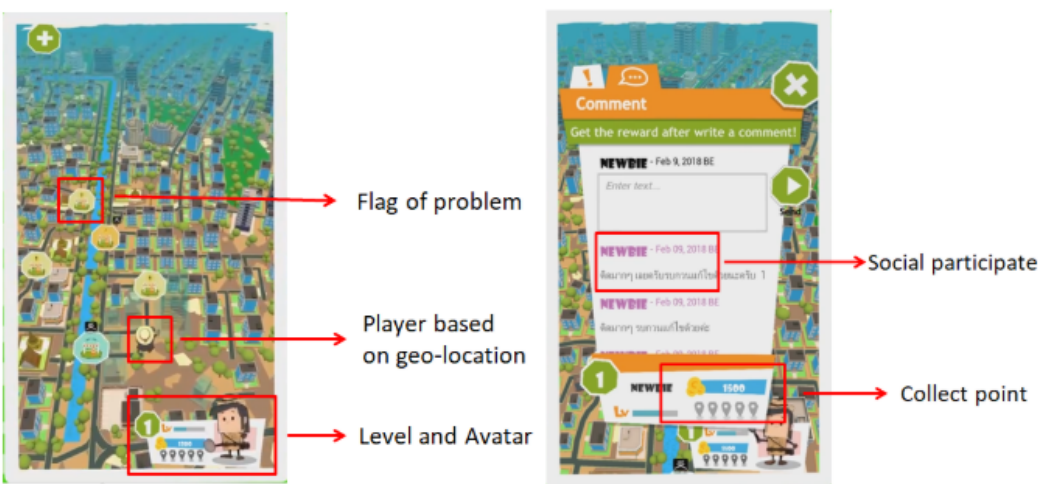

Fig. 4. Game elements implemented in the Mobile application to motivate players.

\subsection{Backend Server}

The purpose of backend in the crowdsourcing system is to support the back-office for the government officer. In terms of management system, we developed a systembased design for corporate management in order to obtain and filter off the citizen's input containing unfavourable opinions. After that, it is stored in a database allowing data analytics. Thus, it consists of three main systems: Game element-based incentives, database and filter request and approve.

\subsection{Real-time cloud-based dashboard}

This real-time cloud-based dashboard uses the report data of the previous 1 hour, collected from the backend server. The government can use it for monitoring the level of problems on the real Chiang Mai city map. The dashboard is overlaid with a number of circles of different sizes and colors where the number in the circle shows the number of case reports from citizens, and the color of circles show the type of problem that we clustered from the whole problem and the size of the circle shows the number of similar problems affected in that area Fig 5.
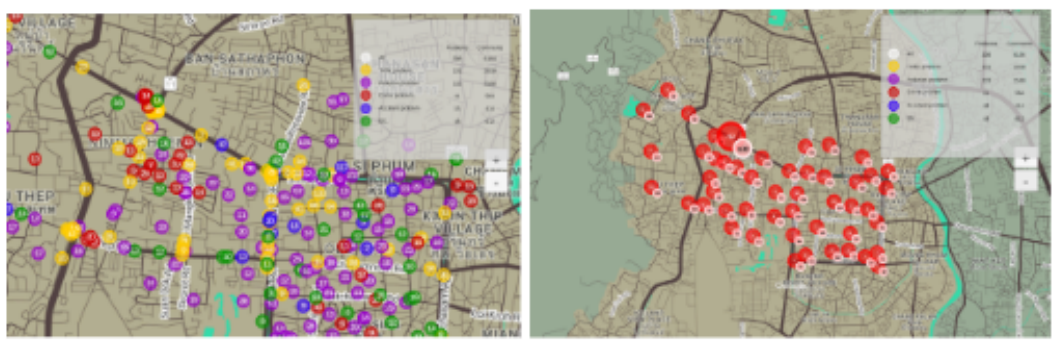

Fig. 5. Shows a real-time dashboard map. The user can click for more details and information. 


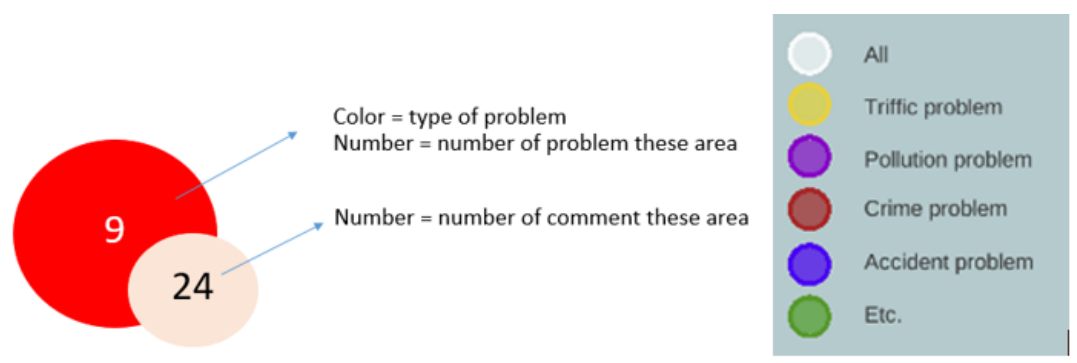

Fig. 6. Shows the meaning of each cluster.

\section{$4 \quad$ Method of Smart Map with Clustering}

When citizens submit or make their complaint via the crowdsourcing system, the system will mark up the point on the map based on their latitude and longitude with tens or hundreds of points per hour Fig 4. However, it can be difficult for the government officer to understand and prioritize the problems in terms of prevention and to propose a solution. In order to solve this problem, we employed the k-mean clustering algorithms [11] to merge each point with the similar scenario together.

\subsection{K-mean algorithms for Crowdsourcing information clustering}

The first k-mean was proposed by MacQuuen [5]. The concepts of algorithms are based on finding a fixed number $(\mathrm{k})$ of clusters in a large set of data. Then, the algorithms are grouped together into a cluster based on the similarities of their attributes and features. Each cluster is defined by a centroid, which is calcuted by the center of data for each cluster. However, in order to address our problem, we decided to use kmean clustering algorithms proposed by Jing et al. [11] The algorithms are modified to add entropy weighting k-means algorithm in order to subspace clustering of highdimensional numeric data. The equation is shown below.

$$
\zeta=\sum_{j=1}^{k}\left[\sum_{i=1}^{n} \sum_{t=1}^{m} \delta_{j i} \lambda_{j t}\left(\left(d_{i t}-C_{j t}\right)\right)^{2}+\gamma \sum_{t=1}^{m} \lambda_{j t} \log \lambda_{j i}\right]
$$

Subject to

$\left\{\sum_{j=1}^{k} \delta_{j i}=1,1 \leq i \leq n, 1 \leq j \leq k, \delta_{j i} \in\{0,1\} \sum_{t=1}^{m} \lambda_{j t}=1,1 \leq j \leq k, 1 \leq t \leq\right.$ $\left.m, 0 \leq \lambda_{j t} \leq 1\right\}$

Where

$\delta_{j i}$ is the degree of the membership of $i$ th data object in $j$ th cluster.

$d_{i t}$ is the value of $t$ th attribute value of $i$ th data object.

$\lambda_{j t}$ is the weight of $t$ th attribute to $j$ th cluster.

$C_{j t}$ is the value of $t$ th attribute value of $j$ th cluster object. 
Our input data is given in GPS coordinates (latitude and longitude). However, the algorithms clustered to data are based solely on the euclidean distance between objects using the haversine formula ( korn et al, 2000) to get back to the attribute of clusters of locations that are not closer than 0.5 kilometre from each other and we fixed the parameter $\mathrm{k}=40$. Our result is shown below.
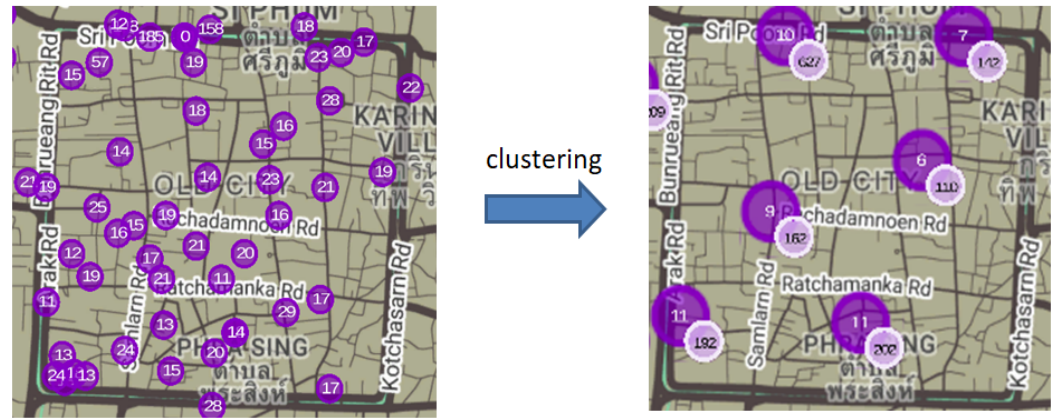

Fig. 7. Result of clustering.

\section{Measurement}

In order to evaluate our proposed Crowdsourcing system, we implemented a system which we tested in the center of Chiang Mai city with the cooperation of Chiang Mai government center and Chiang Mai University School of Public Policy. In technical terms, we developed a mobile application with Unity game engine for cross platforms both ios and android. The Laravel framework is used for the backend server. Unity webgl is used for real-time dashboard. Mysql stored the data in the database. Json is used for data interchange between the Laravel framework and the Mobile application.

For participants, we published the application on Google Play Store and Apple store under the name of "Find issue" in order to let citizens around Chiang Mai download our test beta application which we promoted through social media, advertising boards around Chiang Mai University and via the Center of Chiang Mai. We evaluated our system for 2 months during which 548 interested citizens registered according to our system records. We reviewed the data between April 1and June 1, 2018 having 512 new public problems /complaints from citizens and 6334 comments regarding totally new public problems. The result is shown in Table II.

Table 2. Shows the results of all participants of crowdsourcing

\begin{tabular}{|c|l|c|c|c|c|}
\hline ID & Problems & Problems Statics & Problem (\%) & $\begin{array}{c}\text { Comments } \\
\text { Statistics }\end{array}$ & Comments (\%) \\
\hline 1 & Traffic & 52 & $23.74 \%$ & 2110 & 33.31 \\
\hline 2 & Pollution & 64 & $29.22 \%$ & 2561 & 40.43 \\
\hline 3 & Garbage & 27 & $12.33 \%$ & 564 & 8.9 \\
\hline 4 & Accident & 46 & $21.00 \%$ & 487 & 7.69 \\
\hline 5 & Etc. & 30 & $13.70 \%$ & 612 & 9.66 \\
\hline
\end{tabular}




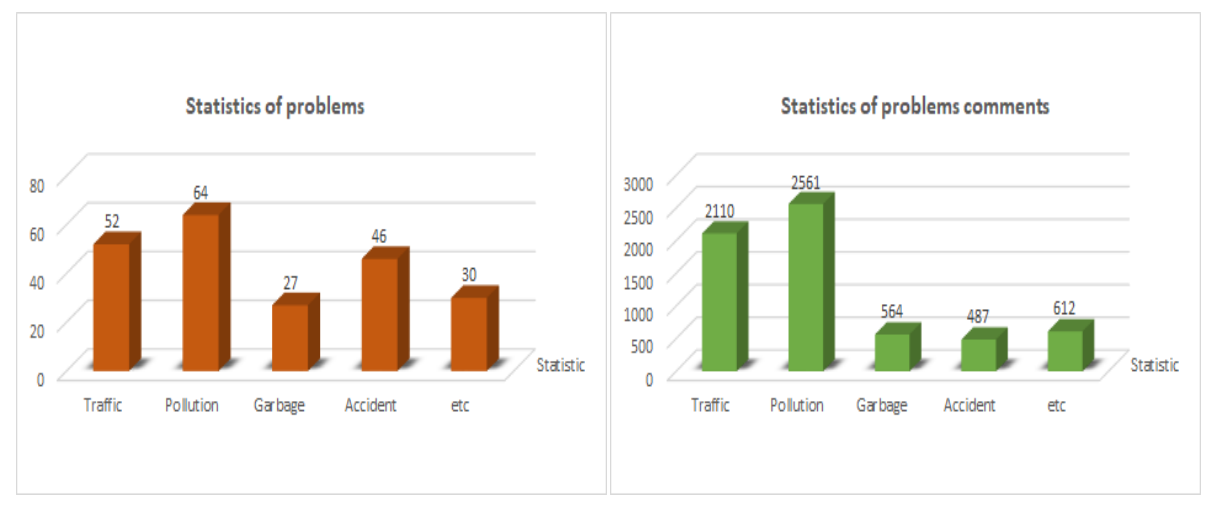

(a) Statistics of problems

(b) Statistics of comments on problems

Fig. 8. Statistics of citizen participation.

Figure 8 shows the contribution of each urban problem to the total information gathered from the crowdsourcing system. There are 5 types of urban problems, and the rates of the 5 types of urban problems in Chiang Mai are:

1) Traffic $(23.74 \%)$

2) Pollution $(29.22 \%)$

3) Garbage (12.33\%)

4) Accidents $(21.00 \%)$

5) Other type of problem (13.70\%)

Comparing the proportion of total urban problems and the number of comments from citizens on each sector type (Figure 8), it can be seen that Pollution problem related complaints account for around $29.22 \%$ of the total problems, exceeding Traffic, Garbage and Accident, as well as other types of problems, with the percentages of $23.74 \%, 21.00 \%, 13.70$ and $12.33 \%$, respectively.

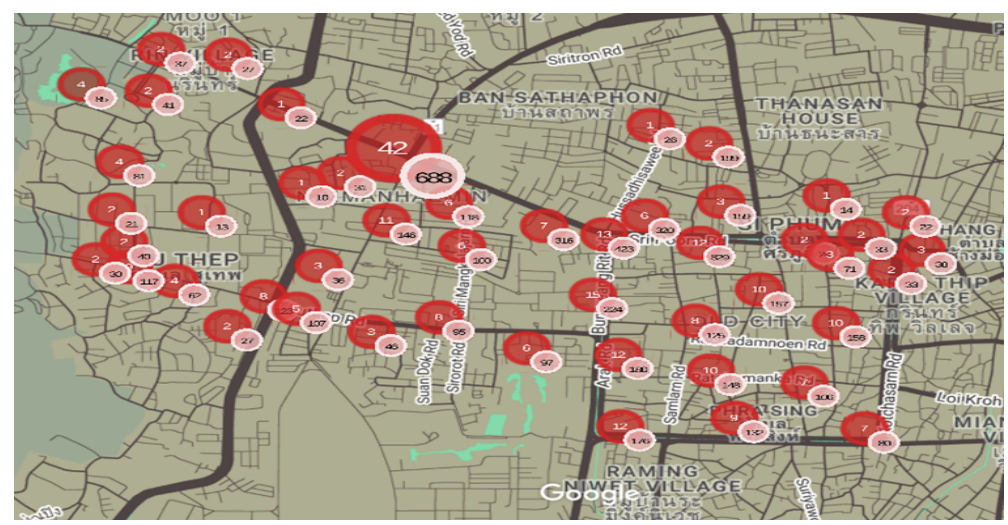

Fig. 9. Real-time dashboard visualization of Chiang Mai city. 


\section{Conclusion}

We proposed a Gamified crowdsourcing system for smart urban government used in Chiang Mai city. Our framework is comprised of the Gamified Crowdsourcing mobile application, game elements based on incentives, a database and filter off data for the backend system. We also developed real-time cloud-based dashboard for government officers for monitoring.

In order to evaluate our proposed system, we have implemented a beta test of application between April 1 and June 1, 2018 which had 512 new public problems and 6334 comments from citizens related to certain problems of the biggest urban problems during that time were pollution and traffic jam with citizens requesting the government officers to solve them.

For the future, we plan to improve and add more complexity to the game elements in order to engage citizens in the use of our system because the high number of participants is the key of successful crowdsourcing systems.

A further suggestion is to plan a modeling algorithm with topic prediction algorithm in order to forecast the next possible urban problem from previous statistics data. Finally, our research framework in crowdsourcing applied to smart governance of in smart city could help in gathering information and boosting up for the research on the smart city domain in the future.

\section{Acknowledgement}

This work was supported by knowledge of innovation center (KIC) Chiang Mai University and Chiang Mai University School of Public Policy.

\section{References}

[1] A. Ojo, E. Curry, and T. Janowski, "Designing Next Generation Smart City Initiatives harbessing Findings and Lessons from a Study of 10 Smart City programs," In 22nd European Conference on Information Systems (ECIS 2014), vol. 2050, pp. 1-14, 2014. https:// doi.org/10.1007/978-3-319-03167-5 4

[2] D. Geiger, and M. Schader, "Personalized task recommendation in crowdsourcing information systems - Current state of the art," Decision Support Systems, vol. 65, pp. 3-16, 2014. https://doi.org/10.1016/j.dss.2014.05.007

[3] D. Puiu, P. Barnaghi, R. Tönjes, D. Kümper, M. I. Ali, A. Mileo,... and F. Gao, "Citypulse: Large scale data analytics framework for smart cities," IEEE Access, vol. 4, pp. 1086-1108, 2016. https://doi.org/10.1109/access.2016.2541999

[4] G. A. Korn, and T. M. Korn, Plane and Spherical Trigonometry: Formulas Expressed in Terms of the Haversine Function. Mathematical Handbook For Scientists: Definitions, Theorems, and Formulas for Reference and Review. New York: McGraw-Hill, 2000.

[5] J. B. MacQuuen, "Some methods for classification and analysis of multivariate observation," In Proc. 5th Berkley Symposium on Mathematical Statistics and Probability, 1967, pp. 281-297.

[6] J. H. Jung, C. Schneider, and J. Valacich, "Enhancing the motivational affordance of information systems: The effects of real-time performance feedback and goal setting in 
group collaboration environments," Management science, vol. 56, no. 4, pp. 724-742, 2010. https://doi.org/10.1287/mnsc.1090.1129

[7] J. Prpić, P. Shukla, J. Kietzmann, and I. McCarthy, "How to Work a Crowd: Developing Crowd Capital through Crowdsourcing," Business Horizons, vol. 8, no. 1, pp. 77-85, 2015. https://doi.org/10.1016/j.bushor.2014.09.005

[8] K. Huotari, and J. Hamari, "Defining gamification: a service marketing perspective," In Proceeding of the 16th international academic MindTrek conference, 2012, pp. 17-22. https://doi.org/10.1145/2393132.2393137

[9] K. Huotari, and J. Hamari, "A definition for gamification: anchoring gamification in the service marketing literature," Electronic Markets, vol. 27, no. 1, pp. 21-31. 2017. https://d oi.org/10.1007/s12525-015-0212-z

[10] K. Robson, K. Plangger, J. H. Kietzmann, I. McCarthy, and L. Pitt, "Is it all a game? Understanding the principles of gamification. Business Horizons," vol. 58, no. 4, pp. 411-420, 2015. https://doi.org/10.1016/j.bushor.2015.03.006

[11] L. Jing, M. K. Ng, and J. Z. Huang, "An entropy weighting k-means algorithm for subspace clustering of high-dimensional sparse data," IEEE Transactions on Knowledge and Data Engineering, vol. 19, no. 8, pp. 1026-1041, 2007. https://doi.org/10.1109/tkde.2007. 1048

[12] M. Claypole, "Learning through crowdsourcing is deaf to the language challenge." The Guardian, 2012

[13] N, Kaufmann, T. Schulze, and D. Veit, "More than fun and money. Worker Motivation in Crowdsourcing-A Study on Mechanical Turk." In AMCIS, vol. 11, no. 2011, pp. 1-11, 2011.

[14] S. Goto, D. Lin, and T. Ishida, "Crowdsourcing for Evaluating Machine Translation Quality,” In LREC, Vol. 2014, May., pp. 345-346, 2014.

[15] S. H. Supangkat, A. A. Arman, R. A. Nugraha, and Y. A. Fatimah, "The Implementation of Garuda Smart City Framework for Smart City Readiness Mapping in Indonesia,” Journal of Asia Paific Studies, Waseda University, vol. 32, pp. 169-176, 2018.

[16] U. Aguilera, O. Peña, O. Belmonte, and D. López-de-Ipiña, "Citizen-centric data services for smarter cities”. Future Generation Computer Systems, vol. 76, pp. 234-247, 2017. https ://doi.org/10.1016/j.future.2016.10.031

[17] Y. C. Zhao, and Q. Zhu, "Effects of extrinsic and intrinsic motivation on participation in crowdsourcing contest," Online Information Review, vol. 38, no. 7, pp. 896, 2014. https:// doi.org/10.1108/oir-08-2014-0188

[18] Werbach, K., \& Hunter, D. (2015). The gamification toolkit: dynamics, mechanics, and components for the win. Wharton Digital Press.

[19] Zichermann, G., \& Cunningham, C. (2011). Gamification by design: Implementing game mechanics in web and mobile apps. " O'Reilly Media, Inc.".

\section{$9 \quad$ Author}

Kitti Puritat is the lecturer in department of Library and Information Science, Chiang Mai University, Thailand. He graduated bachelor and master of computer engineering and $\mathrm{PhD}$. in Knowledge management. He also works as game-based application, knowledge management, information technology, computer vision and artificial intelligence.

Article submitted 2019-06-03. Resubmitted 2019-09-02. Final acceptance 2019-09-15. Final version published as submitted by the authors. 